\title{
Calidad de vida, factores salutogénicos e ideación suicida en adolescentes
}

\author{
Quality of life, salutogenic factors and suicidal ideation in adolescents
}

\author{
Japcy Margarita Quiceno \\ Stefano Vinaccia \\ Universidad de San Buenaventura, Bogotá \\ Grupo Fundación Flor de Liz, Bogotá, Colombia
}

(Rec: 06 de mayo de 2012 / Acept: 11 de julio de 2012)

\begin{abstract}
Resumen
Este estudio tuvo como objetivo evaluar las relaciones de la orientación positiva hacia el futuro de resiliencia, la felicidad y la ideación suicida sobre la calidad de vida de 291 adolescentes de colegios públicos de la ciudad de Bogotá, Colombia. Diseño: correlacional ex post facto, transversal. Instrumentos: dimensión orientación positiva hacia el futuro de la escala de resiliencia ARS, escala de ideación suicida Positiva y Negativa PANSI, ítem de felicidad, y cuestionario KIDSCREEN-52. El análisis de regresión lineal múltiple mostró que la orientación positiva hacia el futuro, las ideas positivas frente a la vida y la felicidad tuvieron un peso significativo sobre el bienestar psicológico y la relación padres de calidad de vida. En conclusión, los adolescentes que tienen metas claras respecto a su futuro, que se sienten felices y valoran y le dan significado a la vida perciben un mejor bienestar psicológico y relación con sus padres.

Palabras clave: calidad de vida, resiliencia, orientación positiva hacia el futuro, felicidad, ideación suicida, adolescencia.
\end{abstract}

\begin{abstract}
This study had with aim to evaluate the relationship of positive orientation towards the future of resilience, happiness and suicidal ideation on the health-related quality of life in 291 adolescents from public schools in Bogotá, Colombia. Design: correlational ex post facto cross. Instruments: positive dimension to the future orientation of the resilience scale ARS, the Positive and Negative Suicidal Ideation Inventory PANSI, item of happiness, and KIDSCREEN-52. The multiple linear regression analysis showed that the positive orientation toward the future, positive ideas towards life and happiness had a significant weight on the psychological and relationship of parent-related quality of life. Conclusion, adolescents who have clear goals about their future, are happier and give you more value and meaning to life perceived better psychological well-being and relationship with parents.

Key words: quality of life, resilience, positive orientation toward the future, happiness, suicidal ideation, adolescence
\end{abstract}

\section{Introducción}

Desde los años 50s (s. XX) hasta la fecha se ha venido estudiando el constructo de resiliencia en niños y adolescentes definiéndosele como la habilidad de luchar y madurar en un contexto cuando hay circunstancias adversas u obstáculos (Rouse y Ingersoll, 1998) o la capacidad de responder y desarrollarse ante las experiencias de eventos vitales estresantes (Markstrom, Marshall y Tryon, 2000).
En un principio el foco de los estudios se centraron en la paradoja de niños y adolescentes que a pesar de vivir en condiciones de vulnerabilidad (pobreza extrema, abandono, familias disfuncionales, psicopatología de los padres, etc.) salían adelante y eran luego adultos competentes y exitosos (Rutter, 1986, 1993, 2006, 2007). Encontrándose que una familia con vínculos de apego seguro, experiencias educativas donde el niño tenga reglas claras, una red de apoyo social y enseñanza eficaz como una comunidad que brinde

Correspondencia: Japcy Margarita Quiceno, Facultad de Psicología, Universidad de San Buenaventura, Cra. 8H No. 72-20, Bogotá, Colombia, e-mail: japcyps@hotmail.com

Stefano Vinaccia, Facultad de Psicología, Universidad de San Buenaventura, Cra. 8H No.72-20, Bogotá, Colombia, email: vinalpi47@hotmail.com 
conexiones sociales y apertura a la comunicación serian promotores de la resiliencia (Earvolino-Ramírez, 2007; Pan y Chan, 2007; Walsh, 2004).

Además los estudios en resiliencia han sido consistentes en plantear como características individuales de los niños y adolescentes resilientes la habilidad en la resolución de problemas, el afrontamiento activo, la autoeficacia, la autoestima, el control emocional, la autonomía e independencia, la empatía, la capacidad para encontrar un significado y propósito en la vida y en el futuro y el sentido del humor (Restrepo, Vinaccia y Quiceno, 2012).

Por otro lado investigadores como Fredrickson (2009) han planteado que cuanto más emociones positivas se experimenta más crecen los niveles de resiliencia. Al respecto Cohn, Fredrickson, Brown, Mikels y Conway (2009) en sus estudios han hallado que la resiliencia está mediada por la relación entre las emociones positivas y el aumento de satisfacción por la vida, lo que sugiere que las personas felices se hacen más satisfechas no sólo porque se sienten mejor, sino porque desarrollan recursos para vivir bien. Además que la felicidad está constituida por un conjunto de elementos como satisfacción por la vida, las estrategias de afrontamiento y las emociones positivas que predicen resultados positivos en diferentes escenarios de la vida. Según Palomera (2009) "la felicidad está constituida por tres dimensiones: emociones positivas, emociones negativas y satisfacción vital. La felicidad es el resultado del grado en que las experiencias de afectividad positiva superan las experiencias de afectividad negativa y del grado en el que una persona percibe que sus metas y motivaciones están resueltas" (p. 273).

Al respecto en diferentes estudios con muestras de adolescentes donde se ha medido los niveles de felicidad y bienestar subjetivo no se ha encontrado diferencias en estas variables en relación a las investigaciones desarrolladas con muestras de adultos (Hernangómez, Vázquez y Hervás, 2009). Ahora bien, los estudios enfatizan que especialmente la familia y la escuela son los principales estamentos educativos que promueven la felicidad en los niños/adolescentes (Palomera, 2009). Entre las características comunes de estos dos estamentos que la promueven, se encuentran: el reforzamiento positivo de todas las actividades y conductas creativas, pro sociales, virtuosas y morales, el desarrollo de estilos de vida saludables y de la autonomía, un adecuado uso del lenguaje hacia si mismo y los demás, focalización de la atención y escucha al niño/adolescente de sus necesidades y expectativas, espacios de flujo donde se desarrolle el juego y tener un ambiente de seguridad y de buenos modelos de vida (Seligman, 2002). Siguiendo por esta línea Holder y Coleman (2009) hallaron en un estudio con niños entre 9 a 12 años asociaciones positivas entre felicidad y las relaciones con la familia y sus pares. Especialmente son más felices aquellos niños que perciben que son valorados e importantes en su hogar y tienen la oportunidad de visitar a sus amigos y ser aceptados por estos, por el contrario, aminora la felicidad los conflictos con compañeros y el mal comportamiento con los demás y la familia. También la felicidad es totalmente independientemente de variables sociodemográficas como la edad y el estado civil de los padres y el número de hermanos. Estos resultados son similares a lo encontrado en estudios con adultos y adolescentes. Especialmente las relaciones sociales fue el factor más importante como predictor de la felicidad.

Por otro lado, también se ha planteado que los adolescentes con bajos niveles de felicidad y perturbación emocional están más propensos a la ideación suicida (Wang, Hughes, Tomblin, Rigby y Langille, 2003). La ideación suicida consiste en pensamientos que tienen las personas de la propia muerte. Estos puede variar en gravedad dependiendo de la especificidad de planes de suicidio y el grado de intención suicida (American Psychiatric Association, 2003). La ideación suicida es una condición necesaria pero no suficiente para desarrollar un comportamiento suicida (Sánchez, Cáceres y Gómez, 2002), ya que para llegar al intento o el suicidio consumado como amenaza para la vida se requieren pensamientos recurrentes, intensos y sistemáticos sobre la muerte y la inutilidad de la vida (VillalobosGalvis, 2010). Respecto a las tasas de ideación suicida de acuerdo al sexo las mujeres presentan niveles mayores en relación a los hombres, pero son los hombres quienes en un porcentaje mayor llevan a cabo el acto suicida (Sánchez et al., 2002). Villalobos-Galvis (2010) desarrolló un estudio con una muestra de 640 estudiantes de la ciudad de Pasto, Colombia con el cuestionario PANSI donde halló que un $20 \%$ de los participantes presentó pensamientos suicidas.

Los factores salutogénicos como resiliencia, felicidad y patogénicos como ideación suicida afectan la calidad de vida infantil (CVI), que hace referencia a "la percepción del bienestar físico, psicológico y social del niño(a) y adolescente dentro de un contexto cultural específico de acuerdo a su desarrollo evolutivo y sus diferencias individuales" (Quiceno, 2007; Quiceno y Vinaccia, 2008). Al respecto Reed, Bell y Edwards (2011) en Estados Unidos realizaron un estudio con 10.606 estudiantes hijos de militares con el objetivo de evaluar las relaciones entre el servicio militar de los padres y la calidad vida, los pensamientos suicidas y la depresión. Se halló que las estudiantes de octavo grado tenían mayor probabilidad de pensamientos suicidas y baja calidad de 
vida a diferencia de los estudiantes varones que tenían solo pensamientos suicidas. Mientras que en los grados décimo al undécimo la calidad de vida, los pensamientos suicidas y el estado de ánimo era más desfavorable en los adolescentes varones en relación con la dedicación de los padres. Por otro lado, Fan et al. (2008) desarrollaron un estudio en China con 3.517 estudiantes universitarios donde evaluaron ideación suicida, calidad de vida, eventos vitales, apoyo social y características sociodemográficas. Se encontró una tasa de $14.1 \%$ de ideación suicida en toda la muestra. Respecto a las mujeres esta fue más alta alcanzando un $15.96 \%$, que era todavía más elevada si la estudiante era madre soltera $(23.79 \%)$ o tenía discapacidad $(25.00 \%)$. Los factores de riesgo asociados con ideación suicida fueron bajos niveles de funcionamiento psicológico, de apoyo familiar y social, pobre disponibilidad de apoyo, bajos recursos materiales y eventos vitales estresantes negativos.

Retomando todo lo anterior y dada la escasez de estudios que relacionen las variables salutogénicas y patogénicas sobre la calidad de vida en el contexto colombiano se diseña un estudio de corte correlacional ex post facto, transversal, con el objetivo de evaluar las relaciones de la orientación positiva hacia el futuro de resiliencia, la felicidad y la ideación suicida sobre la calidad de vida infantil en adolescentes de colegios públicos de la ciudad de Bogotá, Colombia.

\section{Método}

\section{Participantes}

Participaron en este estudio de manera libre y voluntaria 291 adolescentes hombres y mujeres con edades entre los 12 a 16 años de 29 colegios públicos de la ciudad de Bogotá, Colombia de los grados sextos a undécimo escogidos mediante muestreo no aleatorio de sujetos disponibles (véase Tabla 1.).

\section{Instrumentos}

Escala de resiliencia adolescente (Adolescent Resilience Scale, ARS): desarrollado por Oshio, Kaneko, Nagamine y Nakaya (2003) en Japón y traducida al idioma español por Restrepo (2009). La escala ARS consta de 21 ítems con un sistema de respuesta tipo Likert que oscila entre 1 ("definitivamente si") a 5 ("definitivamente no") y comprende tres dimensiones: búsqueda de la novedad ( 7 ítems), regulación emocional (9 ítems) y orientación positiva al futuro (5 ítems) y una escala total. A mayor puntuación mayor resiliencia. En este estudio se empleó para análisis solamente la dimensión orientación positiva al futuro.

Felicidad-1: para el estudio se retoma una pregunta que mide la percepción que tiene el adolescente de su estado de felicidad actual de Quiceno y Vinaccia (2012). La pregunta ¿Qué tan feliz eres? está diseñada para ser contestada en un formato tipo Likert que va de 1 (Soy muy poco feliz) a 5 puntos (Soy muy feliz). El adolescente marca con una equis el número que considera más adecuado para describir su estado de felicidad.

Escala de ideación suicida Positiva y Negativa (The Positive and Negative Suicide Ideation Inventory, PANSI): desarrollado originalmente por Osman, Gutiérrez, Kopper, Barrios y Chiros (1998). Se cuenta con la versión colombiana realizada por Villalobos-Galvis (2010). Esta escala cuenta con 14 ítems con un sistema de respuesta tipo Likert en una escala de 5 puntos que va de 0 (nunca) a 4 (siempre) y dos dimensiones que son ideación suicida positiva que hace referencia a los factores protectores (ítems 6) e ideación suicida negativa que hace alusión a los factores de riesgo (items 8) y una escala total.

Cuestionario de salud y bienestar niños(as) y adolescentes (The KIDSCREEN-52): la versión original fue desarrollada por Herdman y el grupo europeo KIDSCREEN (2002), se cuenta con la versión colombiana (Colombia KIDSCREEN-52) desarrollada por Jaimes (2008). Este cuestionario comprende diez dimensiones que tienen 52 ítems en una escala respuesta tipo Likert con cinco alternativas de respuesta ("nunca" a "siempre") para las dimensiones: bienestar psicológico, estado de ánimo, autopercepción, autonomía, relación padres/vida familiar, recursos económico, apoyo social/pares, entorno escolar y aceptación social (bullying). Mientras que la dimensión bienestar físico tiene un sistema de respuesta tipo Likert con cinco alternativas de respuesta que va de "excelente a "mala".

\section{Procedimiento}

Este estudio fue dirigido por dos psicólogos clínicos y de la salud de la Universidad de San Buenaventura sede Bogotá quienes se encargaron de la idea de investigación, guiar todo el estudio, sistematizar y analizar la información y realizar y presentar los informes de investigación respectivos como el artículo de publicación.

Por otro lado, los representantes legales de la Fundación Flor de Liz de Bogotá fueron los encargados de solicitar a las instituciones educativas los respectivos permisos para la captación de la muestra de estudio, y los psicólogos de 
dicha institución se encargaron de la aplicación de los instrumentos psicométricos. Para el proceso de aplicación de los cuestionarios y la ficha de datos sociodemográficos se tuvo en cuenta previamente los consentimientos informados de los padres de familia y el asentimiento informado de cada adolescente quienes de manera libre y voluntaria decidieran participar en la investigación. El proceso de aplicación se realizó en las instalaciones de la caja de compensación familiar COMPENSAR de Bogotá, en grupos de 20 niños quienes eran acompañados por un psicólogo. El proceso de aplicación tuvo una duración de un mes.

\section{Resultados}

En el siguiente apartado se indican los análisis estadísticos de la investigación. Para el análisis descriptivo de las variables del estudio como edad, orientación positiva al futuro de resiliencia, felicidad, ideación suicida y calidad de vida infantil, se utilizó como medida de tendencia central la media. Así mismo, se calculó la desviación típica (D.T) y las puntuaciones mínimas (Mín.) y máximos (Máx.) de las diferentes dimensiones de los instrumentos psicométricos utilizados. Y respecto a las variables nominales (sexo, escolaridad, con quien vive el adolescente y tipo de enfermedad), discretas (número de hermanos) y continuas (edad) se emplearon porcentajes. Se calculó además el coeficiente alfa de Cronbach para el análisis de fiabilidad de los instrumentos psicométricos del estudio, menos al ítem de felicidad. Para el análisis correlacional de las diferentes dimensiones de los cuestionarios utilizados
(ARS, PANSI, KIDSCREEN-52 e ítem de felicidad) se calculó el Coeficiente $r$ de Pearson. Por último, se empleó un modelo de regresión lineal múltiple para evaluar el peso de las variables predictoras (orientación positiva al futuro de resiliencia, felicidad, ideación suicida) sobre la calidad de vida infantil (variable criterio).

\section{Descripción de la muestra según las características sociodemográficas}

En la Tabla 1 . se puede apreciar que el 59.5\% de la muestra fueron hombres y el $40.5 \%$ mujeres entre los 12 a 16 años de edad (media=14 años). La mayoría de los adolescentes cursaban el grado octavo y noveno de básica secundaria. Se puede evidenciar además que la mayoría viven con sus padres $(\mathrm{n}=277)$, donde el $56.0 \%$ tienen una familia nuclear, el $3.0 \%$ viven con un padrastro o madrastra, el $21.6 \%$ tienen un padre/madre cabeza de familia, el $3.8 \%$ viven con sus padres y abuelos, y el 15.1\% tienen una familia extendida donde el $10.3 \%$ viven con otros familiares (hermanos, abuelos, tíos, primos) y sus padres y el $4.8 \%(\mathrm{n}=14)$ no viven con sus padres si no con abuelos, tíos y hermanos. De otro lado se aprecia que la gran mayoría tienen dos hermanos (33.0\%), seguido de un hermano (29.6\%) y tres hermanos (15.8\%), es de anotar que son pocos los que reportan ser únicos hijos (6.5\%). A nivel de diagnóstico de una enfermedad se observa que una gran proporción informan no tener alguna limitación física (92.8\%) solo el 6.9\% reportan tener enfermedades de tipo reumáticas, gastrointestinales, cardiacas, infecciosas, visuales, neurológicas, ópticas, dermatológicas y hematológicas y una persona no responde $(0.3 \%)$.

Tabla 1. Características sociodemográficas de la muestra $(n=291)$.

\begin{tabular}{|c|c|c|c|c|c|}
\hline & $n$ & $\%$ & & $n$ & $\%$ \\
\hline Sexo & & & Sexo & & \\
\hline Hombre & 173 & $59.5 \%$ & Mujer & 118 & $40.5 \%$ \\
\hline Escolaridad & & & Edad (12 a 16 años) & & \\
\hline Sexto & 43 & $14.8 \%$ & 12 & 49 & 16.8 \\
\hline Séptimo & 55 & $18.9 \%$ & 13 & 52 & 17.9 \\
\hline Octavo & 71 & $24.4 \%$ & 14 & 69 & 23.7 \\
\hline Noveno & 79 & $27.1 \%$ & 15 & 70 & 24.1 \\
\hline Décimo & 31 & $10.7 \%$ & 16 & 51 & 17.5 \\
\hline Undécimo & 12 & $4.1 \%$ & Media (D.T) & 14.0 & $(1.34)$ \\
\hline Con quien vive el adolescente & & & Número de hermanos & & \\
\hline Padres y hermanos & 158 & 54.3 & 0 & 19 & 6.5 \\
\hline Padres y soy único hijo & 5 & 1.7 & 1 & 86 & 29.6 \\
\hline Mamá, padrastro y hermanos & 7 & 2.4 & 2 & 96 & 33.0 \\
\hline Papá, madrastra y hermanos & 1 & .3 & 3 & 46 & 15.8 \\
\hline Papá, madrastra y soy único hijo & 1 & .3 & 4 & 16 & 5.5 \\
\hline Papá y hermanos & 10 & 3.4 & 5 & 15 & 5.2 \\
\hline
\end{tabular}




\begin{tabular}{|c|c|c|c|c|c|}
\hline & $n$ & $\%$ & & $n$ & $\%$ \\
\hline Mamá y hermanos & 44 & 15.1 & 6 & 6 & 2.1 \\
\hline Mamá y soy único hijo & 9 & 3.1 & 7 & 3 & 1.0 \\
\hline Padres, hermanos y abuelos & 11 & 3.8 & 8 & 2 & .7 \\
\hline Familia extendida con padres & 30 & 10.3 & & & \\
\hline Familiares (sin padres, abuelos, tíos, hermanos) & 14 & 4.8 & & & \\
\hline No informa & 1 & .3 & & & \\
\hline Tipo de enfermedad & & & Tipo de enfermedad & & \\
\hline Reumáticas & 1 & .3 & Osteocondromatosis & 1 & .3 \\
\hline Queloides & 1 & .3 & Rinitis & 1 & .3 \\
\hline Arritmia Cardiaca & 1 & .3 & Astigmatismo & 1 & .3 \\
\hline Hipoglicemia & 2 & .7 & Colon Irritable & 1 & .3 \\
\hline Miopia & 3 & 1.0 & Epilepsia & 1 & .3 \\
\hline Dermatitis Atópica & 1 & .3 & & & \\
\hline Taticardia & 2 & .7 & Sin responder & 1 & .3 \\
\hline Otitis & 1 & .3 & Total con diagnóstico & 20 & 6.9 \\
\hline Asma & 3 & 1.0 & Total sin diagnóstico & 270 & 92.8 \\
\hline
\end{tabular}

Nota. $n=$ número de participantes; D.T $=$ Desviación Típica

\section{Análisis descriptivo según las variables psicológicas del estudio}

Se presenta en la Tabla 2. los datos descriptivos de las variables psicológicas y el alfa de Cronbach de los instrumentos empleados. Hallándose a nivel general de aceptable a muy buena fiabilidad en las diferentes dimensiones de los instrumentos psicométricos utilizados en el estudio. La dimensión de orientación positiva al futuro de resiliencia tuvo puntuaciones muy altas, igualmente que el ítem de felicidad. Respecto a la escala de ideación suicida total se aprecian bajos niveles. Y de acuerdo a la calidad de vida infantil se observa niveles medio altos en la dimensión bienestar psicológico; niveles medios en las dimensiones autonomía, relación padres/vida familiar, autopercepción, entorno escolar, bienestar físico y apoyo social/pares; y niveles medio bajos en las dimensiones aceptación social (bullying), estado de ánimo y recursos económicos.

Tabla 2. Estadísticos descriptivos de las variables psicológicas del estudio.

\begin{tabular}{lccccc}
\hline & Media & D.T. & Mínimo & Máximo & Alfa \\
\hline Escala ARS & & & & & \\
Orientación positiva al futuro & 4.75 & 0.51 & 2 & 5 & .84 \\
Escala PANSI & & & & & \\
Ideas positivas & 4.7 & 3.7 & 0 & 22 & .66 \\
Ideas negativas & 3.4 & 5.1 & 0 & 31 & .84 \\
PANSI total & 5.4 & 5.4 & 1 & 34 & .77 \\
& & & & & \\
Felicidad-1 & 4.2 & 0.7 & 1 & 5 & \\
& & & & & \\
Escala KIDSCREEN-52 & & & & & \\
Bienestar físico & 53.7 & 10.4 & 31 & 71 & .80 \\
Bienestar psicológico & 58.1 & 10.0 & 28 & 70 & .84 \\
Estado de ánimo & 43.2 & 12.3 & 6 & 71 & .86 \\
Autopercepción & 50.7 & 10.8 & 27 & 71 & .65 \\
Autonomía & 49.0 & 11.2 & 16 & 68 & .81 \\
Relación con padres y vida familiar & 50.4 & 14.0 & 6 & 69 & .91 \\
Recursos económicos & 43.9 & 9.4 & 24 & 65 & .86 \\
Amigos y apoyo social & 55.4 & 11.2 & 8 & 73 & .85 \\
Ambiente escolar & 51.8 & 9.6 & 26 & 73 & .76 \\
Aceptación social (bullying) & 40.8 & 12.3 & 3 & 59 & .70 \\
\hline
\end{tabular}

Nota. D.T = Desviación Típica 


\section{Análisis de correlación}

A nivel del análisis de correlación de Pearson se observa una asociación positiva y moderada entre la escala PANSI ideas positivas con la dimensión de calidad de vida infantil bienestar psicológico (.412) y una correlación negativa pero débil entre el PANSI ideas negativas con la dimensión relación padres/vida familiar de calidad de vida infantil (-.363). De otro lado se aprecia que el ítem de felicidad tuvo asociaciones positivas y moderadas con las dimensiones bienestar psicológico (.506) y relación padres/vida familiar (.438) de la escala de calidad de vida infantil.

Tabla 3. Análisis de correlación de Pearson de las variables del estudio.

\begin{tabular}{lcc}
\hline & \multicolumn{2}{c}{ Calidad de vida infantil } \\
\hline & $\begin{array}{c}\text { Bienestar } \\
\text { psicológico }\end{array}$ & $\begin{array}{c}\text { Relación padres/ } \\
\text { vida familiar }\end{array}$ \\
\hline Ideas - Positivas - PANSI & $.412^{* *}$ & \\
Ideas - Negativas - PANSI & & $-.363^{* *}$ \\
Ítem Felicidad & $.506^{* *}$ & $.438^{* *}$ \\
\hline
\end{tabular}

\section{Análisis de regresión lineal múltiple}

Respecto a los modelos finales de regresión lineal múltiple se encontró que la dimensión bienestar psicológico de calidad de vida infantil presentó un coeficiente de determinación $\mathrm{R}^{2} \mathrm{c}=41 \%$; explicado positivamente por el ítem de felicidad $(B=0.349 ; t=7.212 ; p=.000)$ e ideas positivas del PANSI $(B=0.244 ; t=5.039 ; p=.000)$, y negativamente por ideas negativas del PANSI $(B=-0,236 ; t=-5.027 ; p=.000) \mathrm{y}$ positivamente por la dimensión orientación positiva al futuro de resiliencia $(B=0.159 ; t=3.303 ; p=.001)$.

De igual modo se aprecia que la dimensión relación padres/vida familiar de calidad de vida infantil presentó un coeficiente de determinación $\mathrm{R}^{2} \mathrm{c}=31 \%$; explicado positivamente por el ítem de felicidad $(B=0.311 ; t=5.842$; $p=.000)$ y negativamente por ideas negativas del PANSI $(B=-0,250 ; t=-4.857 ; p=.000)$ y positivamente por la dimensión ideas positivas del PANSI $(B=0.136 ; t=2.554$; $p=.011)$ y orientación positiva al futuro de resiliencia $(B=$ $0.127 ; t=2.401 ; p=.017$ ).

\section{Discusión}

Los participantes de este estudio fueron adolescentes de bachillerato de diferentes colegios públicos de la ciudad de
Bogotá, quienes tenían una media de edad de 14 años, de estrato socioeconómico colombiano medio bajo (niveles 3-1) quienes en su mayoría pertenecían a una familia nuclear, aunque se percibe una tendencia socio-familiar del padre cabeza de familia, en este caso la figura de la madre. La mayoría de los adolescentes de este estudio no presentaron patologías físicas significativas.

Cabe anotar que las escalas utilizadas en esta investigación no son todas aún validadas para el contexto colombiano, véase la escala de resiliencia ARS contándose con algunas caracterizaciones (Restrepo et al., 2012, Quiceno y Vinaccia, 2012) y la escala de calidad de vida KIDSCREEN-52 que tiene una adaptación cultural (Jaimes, 2008), sin embargo permitieron hacer una exploración sobre el comportamiento de las variables en mención para focalizar y redireccionar futuros estudios. Respecto a la escala de ideación suicida PANSI se cuenta con la validación colombiana (VillalobosGalvis, 2010). Ahora bien en este estudio se aprecia niveles de fiabilidad de aceptables a muy buenos en todas las dimensiones de los instrumentos utilizados.

A nivel general se encontró que los estudiantes que participaron en este estudio tuvieron altos niveles de orientación positiva al futuro de resiliencia, es decir, tenían muy claras sus metas y objetivos personales viendo de forma optimista su futuro, igual que en otros estudios colombianos que han tenido una media entre 4.4 a 4.7, véase el estudio de Restrepo et al. (2012) y Quiceno y Vinaccia (2012) y a diferencia del estudio Oshio et al. (2003) donde las puntuaciones son más inferiores que los estudios colombianos (media=3.4). De igual modo los adolescentes reportaron altos niveles en su estado actual de felicidad, indicando presencia de emociones positivas y satisfacción por la vida, lo que es a fin con otros estudios con población adulta colombiana donde se ha indicado que independientemente de los acontecimientos negativos y/o positivos que les acontezcan se perciben y sienten felices (Cruz y Torres, 2006; Sinisterra, 2007) e igualmente es afín a lo planteado por Hernangómez et al. (2009) donde no se perciben diferencias en los niveles de felicidad en los reportes de los adolescentes con respecto a muestras de adultos.

Por otro lado, los resultados de este estudio son similares en cuanto al PANSI ideas negativas con los estudios de Villalobos-Galvis (2010) con población de estudiantes de la ciudad de San Juan de Pasto en Colombia (media $=3.40$ versus 3.22), pero difieren respecto al PANSI ideas positivas siendo en este estudio más bajas las puntuaciones medias (media $=4.67$ versus 7.64 ), de igual modo, en este estudio se aprecia puntuaciones medias más bajas en la escala total del PANSI (media $=5.41$ versus 10.85 ), indicando baja ideación 
suicida en los adolescentes. Analizando los resultados de esta investigación con los puntos percentiles del estudio de Villalobos-Galvis (2010) en población colombiana se aprecia que un $14.8 \%(n=43)$ de los adolescentes contemplaron al suicidio como un medio para resolver una serie de situaciones que les acontecen y les preocupan. Villalobos-Galvis (2010) plantea que tanto en sus estudios como en otras investigaciones, un $20 \%$ de las muestras poblaciones estudiadas habían tenido igualmente pensamiento de suicidio, y que las ideas suicidas "pueden ser productos cognitivos normales, en el sentido de que un buen porcentaje de personas las tiene a lo largo de su vida. Sin embargo, es la presencia excesiva, intensa, permanente y generalizada la que determina su potencial amenazante" (p. 518).

De igual modo se aprecia en éstos adolescentes una percepción adecuada dentro de los límites normales de acuerdo a la etapa de desarrollo evolutivo, de la calidad de vida infantil, respecto a la autonomía, la relación padres/vida familiar, la autopercepción, el entorno escolar, el bienestar físico y el apoyo social/pares, resultados que son similares a estudios colombianos (Quiceno y Vinaccia, 2012), españoles y chilenos (Tebe et al., 2008; Urzúa, Godoy y Ocay, 2011). Es decir, los adolescentes perciben que están logrando su libertad, autosuficiencia e independencia para tomar decisiones respecto a sus actividades diarias y de su propia vida y que el medio en la medida de sus capacidades les brinda oportunidades para participar en actividades sociales y de ocio. Además reportan un ambiente familiar agradable, de apoyo, amor, entendimiento, disponibilidad y donde son tratados de forma justa. Respecto al entorno escolar estos perciben que tienen las capacidades a nivel cognitivo para aprender y concentrasen, se sienten satisfechos con sus propias habilidades y resultados escolares y perciben el colegio como un lugar agradable donde pueden interactuar con sus profesores. A nivel físico perciben que gozan de buena salud y tiene la habilidad para moverse por la casa y la escuela, para jugar enérgicamente o hacer actividades físicas exigentes como los deportes. Por otro lado, los adolescentes sienten aceptación, apoyo, respeto y confianza por su grupo de amigos, donde pueden interactuar, comunicarse abiertamente y mantener en el tiempo la calidad en sus relaciones.

De igual modo, a nivel de autopercepción, los adolescentes de los colegios públicos de la ciudad de Bogotá perciben que tiene una adecuada autoestima, aceptan su aspecto físico e imagen corporal y tienen seguridad y confianza consigo mismos. Estos resultados son similares a los estudios de Quiceno y Vinaccia (2012) en un colegio privado de la ciudad de Bogotá (media $=50.7$ versus 48.2) y de Tebe et al.
(2008) en España (media $=50.7$ versus 53.2), sin embargo este dato dista del estudio Chileno realizado por Urzúa et al. (2011) donde la percepción de autoaceptación es mucho más desfavorable (media $=50.7$ versus 39.1 ).

De otro lado, son significativos los niveles hallados en la dimensión bienestar psicológico que presentó las puntuaciones más altas entre todas las dimensiones, indicando que estos adolescentes sienten satisfacción por la vida y están teniendo experiencias y emociones positivas como alegría y gozo. Presentándose niveles similares a estudios colombianos véase Quiceno y Vinaccia (2012) (media $=$ 58.1 versus 56.8 ), y puntuaciones más elevadas respecto a estudios iberoamericanos véase España (media $=58.1$ versus 54.4$)$ y Chile (media $=58.1$ versus 53.9$)$ (Tebe et al., 2008; Urzúa et al., 2011).

Sin embargo, paradójicamente, se aprecia que la dimensión estado de ánimo presentó los niveles medios más bajos, indicando cierta presencia de emociones negativas como estrés, depresión, soledad, tristeza, incompetencia y resignación. Es decir, se aprecia en estos adolescentes una ambivalencia emocional. Los resultados de esta investigación son similares a estudios colombianos (media $=43.2$ versus 43.5) (Quiceno y Vinaccia, 2012) pero difieren de estudios españoles como el de Tebe et al. (2008) donde sus niveles son más elevados (media $=43.2$ versus 49.3$)$ y chilenos dónde las puntuaciones son muy bajas (media $=43.2$ versus 23.1) (Urzúa et al., 2011).

De igual modo la dimensión recursos económicos presentó los niveles medios más bajos indicando que estos adolescentes perciben que tienen cierta restricción y desventaja financiera que no les permite tener un estilo de vida comparable a otros adolescentes y que les restringe la oportunidad de hacer y compartir con sus iguales. Estos resultados difieren de investigaciones colombianas (media $=43.9$ versus 51.1$)$ e iberoamericanas como el de España (media $=43.9$ versus 50.7$)$ y Chile (media $=43.9$ versus 48.8) donde se halló un nivel de recursos económicos más favorable (Quiceno y Vinaccia, 2012; Tebe et al., 2008; Urzúa et al., 2011).

Cabe anotar que de todas las dimensiones de calidad de vida la que tuvo los niveles más bajos fue la dimensión aceptación social (bullying), indicando que estos adolescentes perciben que hay uno o varios compañeros que agraden y son irrespetuosos hacia otros que estos consideran más "débiles" (sus víctimas), a quienes atormentan, intimidan, rechazan y les hacen daño a ellos/ellas y a sus objetos personales en repetidas ocasiones, propiciándose así en la víctima sentimientos de ansiedad hacia sus mismos compañero de clase/ curso. $\mathrm{Al}$ respecto en el estudio colombiano (media $=40.8$ 
versus 46.3 ) y de españoles (media $=40.8$ versus 46.0$)$ se halló niveles un poco más favorables de aceptación social con relación a este estudio (Quiceno y Vinaccia, 2012; Tebe et al., 2008), sin embargo en el estudio chileno hubo niveles mucho más inferiores de aceptación social (media $=40.8$ versus 14.2 ) en comparación a los resultados de esta investigación (Urzúa et al., 2011).

Respecto a los análisis de correlación se observa que la percepción de sentirse feliz se asocia positivamente con el bienestar psicológico y satisfacción por la vida familiar y viceversa, por el contrario una percepción desfavorable del ambiente del hogar y la relación con los padres se asocian negativamente con ideas suicidas. Mientras que las ideas positivas hacia la vida tienen una relación directamente proporcional con el bienestar psicológico. Al respecto en estudios desarrollados en China se ha encontrado que los estilos de crianza como la falta de armonía familiar, de comunicación insuficiente, de vinculación afectiva y de cuidado y comprensión de los padres hacia el adolescente y la forma de control son factores relacionadas con la ideación suicida en adolescentes (Chung, Luk y Mak, 1987, Fong, 1993; Lau, 1994). Resultados que no distan mucho de lo reportado en los países occidentales (Lai y McBride-Chang, 2001).

Lo anterior se corrobora en el análisis de regresión lineal múltiple donde el no tener ideas suicidas y tener una orientación optimista al futuro de resiliencia impactan positivamente sobre el bienestar psicológico de calidad de vida a diferencia de las ideas suicidas que influyen negativamente sobre esta dimensión. Además los sentimientos de felicidad, las ideas positivas hacia la vida y el tener a nivel de resiliencia metas claras y optimistas hacia el futuro influyen positivamente sobre la relación padres y la vida familiar de calidad de vida, al contrario las ideas suicidas afectan esta dimensión negativamente. De igual modo en el estudio de Quiceno y Vinaccia (2012) con adolescentes de colegios privados de Bogotá se halló que la resiliencia tenía un peso importante sobre la relación padres y la vida familiar de calidad de vida y la felicidad se asociaba con la resiliencia.

En conclusión, aunque a nivel general no hay indicadores clínicos de ideación suicida y estos adolescentes tiene una orientación positiva al futuro de resiliencia y la calidad de vida es adecuada, si bien, se debe prestar atención al estado de ánimo en esta etapa ya que por un lado se percibe que están satisfechos con la vida pero por otro manifiestan presencia de emociones negativas. De igual modo las manifestaciones de bullying en el entorno escolar ameritan urgentemente atención clínica a nivel psicosocial. Por otro lado se aprecia una vez más que la resiliencia, es decir el tener ideas claras respecto al futuro y verlo de manera optimista, más los sentimientos de felicidad y un adecuado soporte familiar son factores protectores frente a la ideación suicida.

Estos resultados sugieren el desarrollo de programas de intervención focalizados desde la psicología positiva donde se potencie las fortalezas humanas para proteger la salud emocional de los adolescentes. En última instancia, "una mayor sinergia entre la psicología positiva y la salud pública puede ayudar a promover la salud mental de manera innovadora" (Kobau et al., 2011).

\section{Referencias}

American Psychiatric Association - APA (2003). Practice guideline for the assessment and treatment of patients with suicidal behaviors. American Journal of Psychiatry, 160, 1-60.

Cohn, M. A., Fredrickson, B., Brown, S. L., Mikels, J. A., y Conway, A. M. (2009). Happiness unpacked: Positive emotions increase life satisfaction by building resilience. Emotion, 9, 361-368.

Chung, S. Y., Luk, S. L., y Mak, F. (1987). Attempted suicide in children and adolescents in Hong Kong. Social Psychiatry, 22, 102-106.

Cruz, J., y Torres, J. (2006). ¿De qué depende la satisfacción subjetiva de los colombianos? Cuadernos de Economía, 45, 131-154.

Earvolino-Ramírez, M. (2007). Resilience: A Concept Analysis. Nursing Forum, 42, 73-82.

Fan, Y. G., Xiao, Q., Wang, Q., Li, W. X., Dong, M.X., y Ye, D. Q. (2008). Study on the influencing factors related to suicide ideation among undergraduates in Anhui province. Zhonghua Liu Xing Bing Xue Za Zhi, 29, 241-244.

Fong, Y. Y. (1993). A study on suicidal ideation and attempted suicide in 316 secondary school students. Hong Kong Journal of Mental Health, $22,43-49$.

Fredrickson, B. (2009). Vida positiva. Bogotá, Colombia: Norma

Herdman, M., Rajmil, L., Ravens-Sieberer, U., Bullinger, M., Power, M., Alonso, J., y the European KIDSCREEN and DISABKIDS groups (2002). Expert consensus in the development of a European healthrelated quality of life measure for children and adolescents: a Delphi study. Acta Pediatrica, 91, 1385-1390.

Hernangómez, L., Vázquez, C., y Hervás, G. (2009). El paisaje emocional a lo largo de la vida. En C. Vázquez y G. Hervás (Eds.), La ciencia del Bienestar (pp. 143-179). Madrid, España: Alianza

Holder, M., y Coleman, B. (2009). The Contribution of Social Relationships to Children's Happiness. Journal of Happiness Studies, 10, 329-349.

Jaimes, M. L. (2008). Adaptación cultural a la población escolar colombiana del KIDSCREEN y del VSP-A, instrumentos de calidad de vida relacionada con la salud (CVRS) para niños(as) y adolescentes (Tesis doctoral inédita). Universidad de Alicante, España.

Kobau, R., Seligman, M. E., Peterson, C., Diener, E., Zack, M. M.,... Chapman, D. (2011). Mental health promotion in public health: perspectives and strategies from positive psychology. American Journal of Public Health, 101, 1-9.

Lai, K. W., y McBride-Chang, C. (2001). Suicidal ideation, parenting style, and family climate among Hong Kong adolescents. International Journal of Psychology, 36, 81-87.

Lau, W. M. (1994). Fatal suicides among children and adolescents $1992-$ 1994. Bulletin of the Hong Kong Psychological Society, 32/33, 105-112. 
Markstrom, C. A., Marshall, S. K., y Tryon, R. J. (2000). Resiliency, social support, and coping in rural low-income Appalachian adolescents from two racial groups. Journal of Adolescence, 23, 693-703.

Oshio, A., Kaneko, H., Nagamine, S., y Nakaya, M. (2003). Construct validity of the Adolescent Resilience Scale. Psychological Reports, 93, 1217-1222.

Osman, A., Gutiérrez, P., Kopper, B., Barrios, F., y Chiros, C. (1998). The positive and negative suicide ideation inventory: Development and validation. Psychological reports, 82, 783-793.

Palomera, R. (2009). Educando para la felicidad. En E. G. FernándezAbascal (Ed.), Emociones positivas (pp. 247-274). Madrid, España: Pirámide.

Pan, J. Y., y Chan, L. W. (2007). Resilience: A new research area in positive psychology. PSYCHOLOGIA An International Journal of Psychological Sciences, 50, 164-176.

Quiceno, J. M. (2007). Calidad de vida infantil. (Manuscrito no publicado). Universidad Autónoma de Madrid, España

Quiceno, J. M., y Vinaccia, S. (2008). Calidad de vida relacionada con la salud infantil: Una aproximación conceptual. Psicología y Salud, 18, 33-44.

Quiceno, J. M., y Vinaccia, A. (2012). Calidad de vida relacionada con la salud, factores salutogénicos y patogénicos en adolescentes escolarizados de la ciudad de Bogotá. (Investigación en curso). Universidad de San Buenaventura sede Bogotá, Colombia.

Reed, S. C., Bell, J. F., y Edwards, T. C. (2011). Adolescent well-being in Washington state military families. American Journal of Public Health, 101, 1676-1682.

Restrepo, C. (2009). Anteproyecto de investigación: Evaluación de la calidad de vida, la resiliencia y la depresión en adolescentes escolarizados de la ciudad de Medellín (Manuscrito no publicado). Universidad de San Buenaventura seccional Medellín, Colombia.

Restrepo, C., Vinaccia, S., y Quiceno, J. M. (2012). RESILIENCIA Y DEPRESIÓN: Un estudio exploratorio desde la calidad de vida en la adolescencia. Suma Psicológica, 18, 2.
Rouse, K. A., y Ingersoll, G. M. (1998). Longitudinal health endangering behavior risk among resilient and nonresilient early adolescents. Journal of Adolescent Health, 23, 297-302

Rutter, M. (1986). Resilience in the face of adversity: protective factors and resistance to psychiatric disorder. British Journal of Psychiatry, 147, 598-611.

Rutter, M. (1993). Resilience: Some conceptual considerations. Journal of Adolescent Health, 14, 626-631.

Rutter, M. (2006). Implications of Resilience Concepts for Scientific Understanding. Annals New York Academy of Sciences, 1094, 1-12.

Rutter, M. (2007). Resilience, competence and coping. Child Abuse y Neglect, 31, 205-209.

Sánchez, R., Cáceres, H., y Gómez, D. (2002). Ideación suicida en adolescentes universitarios: prevalencia y factores asociados. Biomédica, 22, 407-416.

Seligman, M. E. P. (2002). La auténtica felicidad. Barcelona, España: Vergara

Sinisterra, D. (2007). Colombian happiness: A look at life satisfaction and explanatory style (Disertación Doctoral no publicada). Florida, EEUU: Atlantic University

Tebe, C., Berra, S., Herdman, M., Aymerich, M., Alonso, J., y Rajmil, L. (2008). Fiabilidad y validez de la versión española del KIDSCREEN-52 para población infantil y adolescente. Medicina Clínica, 130, 650-654.

Urzúa, A., Godoy, J., y Ocay, K. (2011). Competencias parentales percibidas y calidad de vida. Revista Chilena de Pediatría, 82, 300-310.

Villalobos-Galvis, F. (2010). Validez y fiabilidad del Inventario de Ideación Suicida Positiva y Negativa - PANSI, en estudiantes colombianos. Universitas Psychologica, 9, 509-520.

Walsh, F. (2004). Resiliencia familiar: Estrategias para su fortalecimiento. Madrid, España: Amorrortu.

Wang, J., Hughes, J., Tomblin, G., Rigby, J. A., y Langille, D. B. (2003). Suicidal Behaviours Among Adolescents in Northern Nova Scotia. Canadian Journal of Public Health, 94, 207-211. 
